\title{
Effects of Hypothyroidism on Quality of Life of Differentiated Thyroid Carcinoma Patients undergoing Postoperative lodine-131 Therapy
}

\author{
YAOYAO WANG, XINGPING SHEN ${ }^{1}$ AND FAN YANG ${ }^{*}$
}

General Practice Department of Hospital in Zhongnan University of Economics and Law, Wuhan, Hubei, ${ }^{1}$ Department of Endocrinology and Metabolism, Zhongshan Hospital Xiamen University, Xiamen, 361004, ${ }^{2}$ Surgery Department of Hospital in Huazhong University of Science \& Technology, Hongshan, Wuhan, Hubei 430074, China

Wang et al.: Effects of Hypothyroidism on Patients undergoing Postoperative Iodine-131 Therapy

To evaluate the effects of hypothyroidism on the quality of life of differentiated thyroid carcinoma patients undergoing postoperative iodine-131 $\left(\mathrm{I}^{131}\right)$ therapy. A total of 140 thyroid carcinoma inpatients treated in our hospital from January 2016 to June 2017 were collected. All patients were treated with postoperative $I^{131}$ therapy, and followed up for 3 years. The effect of $I^{131}$ therapy on hypothyroidism was observed from the perspectives of gender, age, course of disease and $\mathrm{I}^{131}$. On this basis, multivariate logistic regression analysis was performed to screen the influencing factors for hypothyroidism in differentiated thyroid carcinoma patients undergoing postoperative $I^{131}$ therapy, and the predictive model was established. Quality of life of patients was compared before and after $I^{131}$ therapy, and the correlation between thyroid function indices and quality of life was further analyzed. The predictive value of $I^{131}$ therapy after differentiated thyroid carcinoma surgery for the levels of thyroid function indices and quality of life was evaluated using receiver operating characteristic curve. A total of 140 patients undergoing $I^{131}$ therapy after differentiated thyroid carcinoma surgery were enrolled. It was found through follow-up that the incidence rate of hypothyroidism was $35.00 \%(49 / 140)$. The incidence of hypothyroidism after $I^{131}$ therapy was related to the thyroid quality, course of disease, cumulative dose of $I^{131}$ and operation method $(p<0.01)$, but not related with gender, age or pathological type $(\mathrm{p}>\mathbf{0 . 0 5})$. The forest plot of multivariate logistic regression analysis results showed that thyroid quality, course of disease, $I^{131}$ dose and operation method were independent risk factors for hypothyroidism after postoperative $I^{131}$ therapy. Compared with before $I^{131}$ therapy, each index of quality of life was significantly increased in differentiated thyroid carcinoma patients after postoperative $I^{131}$ therapy $(p<0.05)$. The results of correlation analysis revealed that some indices of quality of life (physical function, bodily pain, social function and mental health) were all positively correlated with thyroid function indices total triiodothyronine, free triiodothyronine, free thyroxine, total thyroxine and thyroid stimulating hormone $(\mathbf{p}<\mathbf{0 . 0 5}$ or $\mathbf{p}<\mathbf{0 . 0 1})$. Other indices of quality of life (role physical, general health, vitality and role emotional) had no significant correlations with triiodothyronine, free triiodothyronine, free thyroxine, total thyroxine or thyroid stimulating hormone $(p>0.05)$. The area under receiver operating characteristic curve of thyroid stimulating hormone level in differentiated thyroid carcinoma patients was 0.819 (95\% CI: 0.744-0.893), and the optimal cutoff value was $0.63 \mathrm{mU} / \mathrm{L}$. The cutoff value of $\mathrm{I}^{131}$ dose was 7.35 GBq, and the area under receiver operating characteristic curve was 0.726 (95\% CI: 0.623-0.830). Hypothyroidism in differentiated thyroid carcinoma patients undergoing postoperative $I^{131}$ therapy has a significant impact on quality of life. There are correlations between indices of quality of life and thyroid function indices. Postoperative $I^{131}$ therapy not only mitigates the symptoms of hypothyroidism, but also improves the overall quality of life of patientsr

Key words: Differentiated thyroid carcinoma, hypothyroidism, quality of life, iodine-131 therapy

Since the early $1990 \mathrm{~s}$, the incidence rate of thyroid carcinoma has dramatically risen, and it is particularly common in female cancers. Differentiated thyroid carcinoma (DTC) is a common type of thyroid carcinoma

*Address for correspondence E-mail: 654363803@qq.com

January-February 2021
This is an open access article distributed under the terms of the Creative Commons Attribution-NonCommercial-ShareAlike 3.0 License, which allows others to remix, tweak, and build upon the work non-commercially, as long as the author is credited and the new creations are licensed under the identical terms

Accepted 26 February 2021

Revised 15 December 2020

Received 12 September 2020 Indian J Pharm Sci 2021;83(1):127-133 
in clinical endocrine breast surgery. DTC such as papillary thyroid carcinoma and follicular carcinoma accounts for about $96.40 \%$ of thyroid carcinoma ${ }^{[1]}$. DTC can be surgically resected, and radionuclide iodine therapy and thyroid stimulating hormone (TSH) suppression therapy are effective clinical treatment means, with generally good prognosis ${ }^{[2]}$.

However, metastasis occurs in about 4-9 \% of patient. Iodine-131 $\left(\mathrm{I}^{131}\right)$ therapy is the main therapeutic method for patients with advanced DTC. It has been found that the overall survival rate of patients not treated with $\mathrm{I}^{131}$ is lower than that of patients treated with $\mathrm{I}^{131}$ (10-year survival rate: about $10.02 \%$ vs. $42.20 \%)^{[3]}$. Before postoperative $\mathrm{I}^{131}$ therapy, the patient's thyroid function is in decline. Hypothyroidism causes damage to hepatocytes through damaging mitochondrial membrane lipids, thus leading to various abnormalities in the body, such as dyslipidemia and insulin resistance, and even inducing liver cancer ${ }^{[4-6]} . \mathrm{I}^{131}$ therapy is a hot spot in clinical research currently, which is a widelyused effective adjuvant therapy for thyroid carcinoma. However, the $\beta$ rays of $I^{131}$ therapy may destroy the thyroid follicular cells, enhance their apoptosis and weaken their proliferation ability. As a result, the number of thyroid follicular cells will gradually decline, resulting in inevitable hypothyroidism.

The quality of life (QOL) expressing patients' subjective feelings and the objective indices such as serum thyroid hormone level can be used to evaluate the therapeutic effect on DTC more comprehensively ${ }^{[7]}$. In recent years, tumor-free survival has become an important means of evaluating the therapeutic effect on patients. However, people have recognized that tumor treatment and management can improve QOL of patients. Therefore, the goal of cancer treatment is not only to increase the survival rate, but also to ensure QOL of patients, the latter of which is of great importance. Therefore, relevant issues should be considered from the evaluation of QOL of DTC patients ${ }^{[8]}$. In the present study, the efficacy of postoperative $\mathrm{I}^{131}$ therapy on DTC patients was analyzed, the influencing factors causing hypothyroidism were explored from age, gender, thyroid quality, course of disease and $\mathrm{I}^{131}$ dose, and the correlation between postoperative hypothyroidism and QOL of DTC patients was further detected, so as to provide a theoretical basis for the hospitalization management of patients treated with $\mathrm{I}^{131}$ therapy, guide the development of effective coping strategies, and improve QOL of patients.

\section{MATERIALS AND METHODS}

\section{Subjects:}

A total of 140 thyroid carcinoma inpatients treated in our hospital from January 2016 to June 2017 were collected. All patients underwent thyroid surgery after admission and they were diagnosed as DTC through postoperative pathology and then underwent $\mathrm{I}^{131}$ therapy. There were 51 males and 89 females aged 50.53 y old on average. Related data were inquired in our department and treatment places, and general data and laboratory examination data of patients were obtained. This study was reviewed and approved by the Medical Ethics Committee, and all patients and their families were informed and signed the consent.

\section{Inclusion and exclusion criteria:}

Inclusion criteria were as follows: patients with normal thyroid function before surgery, those undergoing thyroid surgery, those meeting the diagnostic criteria for DTC after surgery and those with solid thyroid space-occupying lesions confirmed by cervical ultrasound, CT and other imaging examinations. Finally, 140 DTC patients with a level of postoperative $\mathrm{TSH}>30 \mathrm{mU} / 1$ were enrolled. Exclusion criteria were as follows: patients with a history of mental disorders or severe heart, liver or kidney diseases or those with hypertension, diabetes mellitus or dyslipidemia.

\section{$I^{131}$ therapy:}

All patients were hospitalized at $7 \mathrm{~d}$ before $\mathrm{I}^{131}$ therapy, during which they were instructed to keep a low-iodine diet and stop taking levothyroxine tablets. After admission, routine blood and urine examinations were performed, and the abdominal and thyroid color Doppler ultrasound examination, thyroid imaging, and detection of thyroid iodine uptake function and serum indices of thyroid function were also conducted. Based on the thyroid quality detected, course of disease and age, oral $\mathrm{I}^{131}$ therapy in different doses was given. After treatment, the patients were regularly followed up for 3 y until June 2020, and the indices of thyroid function and QOL were detected.

\section{Detection of serum thyroid function indices:}

Thyroid function indices were detected via radioimmunoassay, including $\mathrm{TSH}$, total triiodothyronine (TT3), free triiodothyronine (FT3), free thyroxine (FT4), and total thyroxine (TT4). 


\section{Assessment of therapeutic effects:}

Cure: The patients were followed up for more than 6 months, the symptoms and signs of hypothyroidism completely disappeared, and the levels of serum TSH, TT3, FT3, FT4 and TT4 all returned to normal. Improvement: After $\mathrm{I}^{131}$ therapy, the symptoms of hypothyroidism were relieved, the signs partially disappeared, including recurrence, and the levels of serum TSH, TT3, FT3, FT4 and TT4 did not decline or rebounded after decline. Ineffectiveness: After $I^{131}$ therapy, the symptoms of hypothyroidism had no improvement but became worse, and the levels of serum TSH, TT3, FT3, FT4 and TT4 could not decline. Occurrence of hypothyroidism: The patients had symptoms of hypothyroidism, the levels of serum TT3, FT3, FT4 and TT4 declined, while the level of TSH rose, and the symptoms of hypothyroidism were relieved after $\mathrm{I}^{131}$ therapy.

\section{Assessment of health-related QOL:}

The Chinese version of the 36-item Short Form Health Survey (SF-36) questionnaire was used, which was slightly modified ${ }^{[9]} .8$ health-related dimensions were detected: physical function, role physical, bodily pain, general health, vitality, social function, role emotional and mental health. The number of items in each dimension was different, so it was needed to convert the initial score of each dimension into 0-100 points. The higher the score, the better the quality of this dimension. The comprehensive score was given using the SF-36 questionnaire in the predictive model evaluation, and converted into 82-100 points (good), 51-81 points (medium) and $0-50$ points (poor).

\section{Statistical analysis:}

SPSS 25.0 software was used for statistical analysis. Quantitative data were expressed as mean \pm standard deviation. Independent-samples $t$ test was performed for comparison between two groups, and $F$ test was conducted for comparison among groups. Numerical data were expressed as frequency or rate, and $\chi^{2}$ test was performed for intergroup comparison. The curative effect was analyzed using rank sum test. In combination with the clinical factors, variables with statistically significant differences in univariate analysis were used as candidate variables and incorporated into multivariate logistic regression model to establish the predictive model. The predictive value of relevant indices for patients was analyzed using receiver operating characteristic (ROC) curve. $\mathrm{p}<0.05$ was considered to be statistically significant.

\section{RESULTS AND DISCUSSION}

The curative effect on 140 patients with DTC at different time points after postoperative $\mathrm{I}^{131}$ therapy had statistically significant differences $(p<0.05)$. The incidence rate of hypothyroidism was $35.00 \%$ (49/140), and it had statistically significant differences at different time points after $\mathrm{I}^{131}$ therapy $\left(\chi^{2}=39.83\right.$, $\mathrm{p}<0.01)$ (Table 1). The efficacy against hypothyroidism after $I^{131}$ therapy was related to thyroid quality, course of disease, cumulative dose of $\mathrm{I}^{131}$ and operation method $(p<0.01)$. However, the incidence of hypothyroidism after postoperative $\mathrm{I}^{131}$ therapy had no correlation with gender, age or pathological type $(\mathrm{p}>0.05)$ (Table 2$)$.

The influencing factors for hypothyroidism in 140 DTC patients after postoperative $\mathrm{I}^{131}$ therapy were subjected to multivariate analysis. Variables with statistically significant differences in univariate analysis were incorporated into the analysis, with the occurrence of hypothyroidism as the dependent variable. It was found that thyroid quality, course of disease, $\mathrm{I}^{131}$ dose and operation method were independent risk factors for hypothyroidism after postoperative $\mathrm{I}^{131}$ therapy (fig. 1). Compared with before $\mathrm{I}^{131}$ therapy, each index of QOL was significantly increased in DTC patients after postoperative $I^{131}$ therapy $(\mathrm{p}<0.05)$ (Table 3$)$. Some indices of QOL (physical function, bodily pain, social function and mental health) were all positively correlated with thyroid function indices TT3, FT3, FT4, TT4 and TSH $(\mathrm{p}<0.05$ or $\mathrm{p}<0.01)$. Other indices

TABLE 1: FOLLOW-UP RESULTS OF PATIENTS AFTER I131 THERAPY

\begin{tabular}{lccccc}
\hline Follow-up time (year) & $\mathbf{n}$ & Cure (\%) & Improvement (\%) & Ineffectiveness (\%) & $\begin{array}{c}\text { Incidence rate of } \\
\text { hypothyroidism (\%) }\end{array}$ \\
\hline $0 \sim$ & 74 & $52(70.27 \%)$ & $19(25.68 \%)$ & $3(4.05 \%)$ & $41(55.41 \%)$ \\
$1 \sim$ & 38 & $32(84.21 \%)$ & $6(15.79 \%)$ & 0 & $5(13.16 \%)$ \\
$2 \sim$ & 22 & $20(90.91 \%)$ & $2(9.09 \%)$ & 0 & $2(9.09 \%)$ \\
3 & 6 & $6(100.00 \%)$ & 0 & 0 & $1(16.67 \%)$ \\
Total & 140 & $110(78.57 \%)$ & $27(19.29 \%)$ & $3(2.14 \%)$ & $49(35.00 \%)$ \\
\hline
\end{tabular}


TABLE 2: UNIVARIATE ANALYSIS OF EFFICACY ON HYPOTHYROIDISM AFTER I ${ }^{131}$ THERAPY

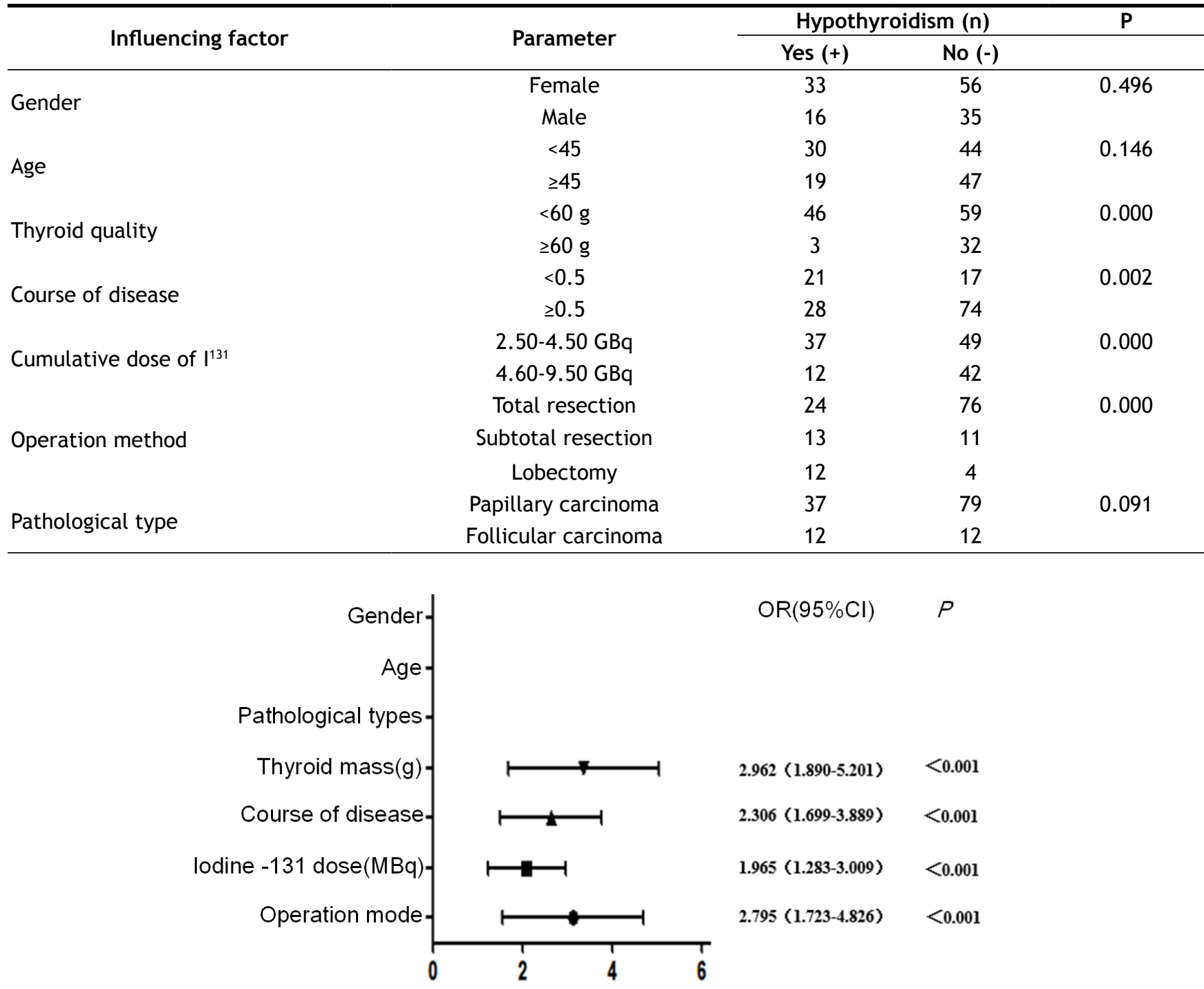

Fig. 1: Forest plot of predictive model of hypothyroidism in DTC patients

TABLE 3: QOL BEFORE AND AFTER I ${ }^{131}$ THERAPY

\begin{tabular}{|c|c|c|c|c|}
\hline Item & Before I $^{131}$ therapy (mean) & After I'131 therapy (mean) & $\bar{t}$ & $\bar{p}$ \\
\hline Physical function & $60.99 \pm 13.06$ & $82.62 \pm 20.13$ & 8.07 & $<0.05$ \\
\hline Role physical & $58.42 \pm 19.64$ & $73.95 \pm 21.17$ & 3.62 & $<0.05$ \\
\hline Bodily pain & $52.98 \pm 18.21$ & $80.01 \pm 22.74$ & 9.65 & $<0.05$ \\
\hline General health & $43.52 \pm 16.97$ & $61.59 \pm 19.28$ & 6.13 & $<0.05$ \\
\hline Vitality & $49.47 \pm 12.35$ & $62.13 \pm 17.35$ & 5.38 & $<0.05$ \\
\hline Social function & $53.36 \pm 20.15$ & $80.00 \pm 20.72$ & 9.14 & $<0.05$ \\
\hline Role emotional & $58.21 \pm 10.83$ & $69.29 \pm 12.30$ & 2.99 & $<0.05$ \\
\hline Mental health & $48.76 \pm 12.51$ & $81.54 \pm 15.89$ & 10.32 & $<0.05$ \\
\hline
\end{tabular}

of QOL (role physical, general health, vitality and role emotional) had no significant correlations with TT3, FT3, FT4, TT4 or TSH (p>0.05) (Table 4).

The median TSH level was $0.26 \mathrm{mU} / 1$ in good QOL group, which was significantly lower than that in medium QOL group $(0.51 \mathrm{mU} / \mathrm{l})(\mathrm{U}=59.0, \mathrm{p}<0.05)$ and poor QOL group $(0.97 \mathrm{mU} / \mathrm{l})(\mathrm{U}=53.0, \mathrm{p}<0.05)$. On this basis, the ROC curves of the relationship between TSH level and QOL of patients was constructed. Evaluation of predictive model: The ROC curves of TSH level in detecting QOL of patients were plotted (fig. 2 and fig. 3). It was found that the area under the ROC curve of TSH level was 0.819 (95\% CI: 0.744-0.893), and the optimal cutoff point was $0.63 \mathrm{mU} / 1$. The diagnostic sensitivity and specificity of TSH were $73.16 \%$ and $82.72 \%$, respectively. The cutoff value of $\mathrm{I}^{131}$ dose was 
$7.35 \mathrm{GBq}$, the area under the ROC curve was 0.726 (95\% CI: 0.623-0.830), and the diagnostic sensitivity and specificity were $71.86 \%$ and $80.07 \%$, respectively.

Among thyroid malignancies, thyroid carcinoma is the most common endocrine carcinoma with an increasing incidence rate in the past few years, and it is also the top five tumors in women. DTC is the main histological subtype, and it has a low mortality rate, but recurrence

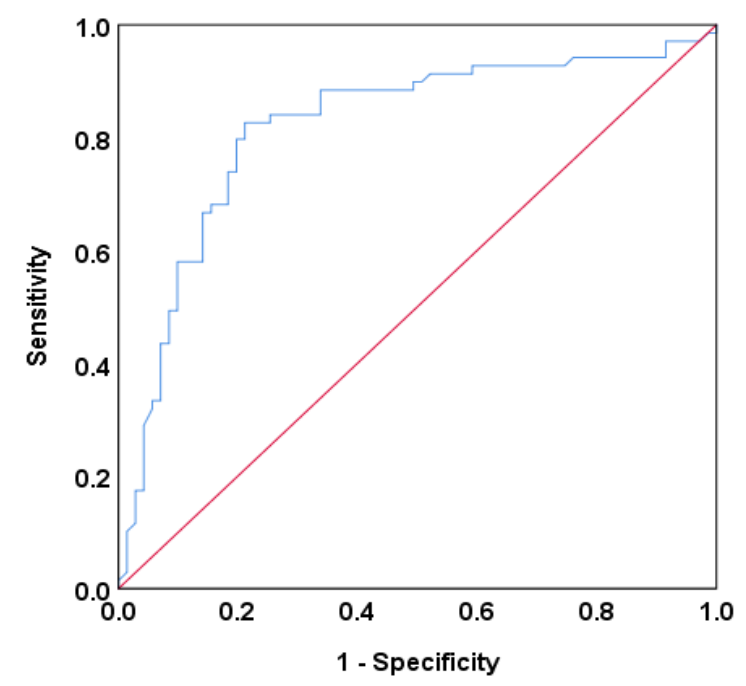

Fig. 2: ROC curve for cutoff value of TSH

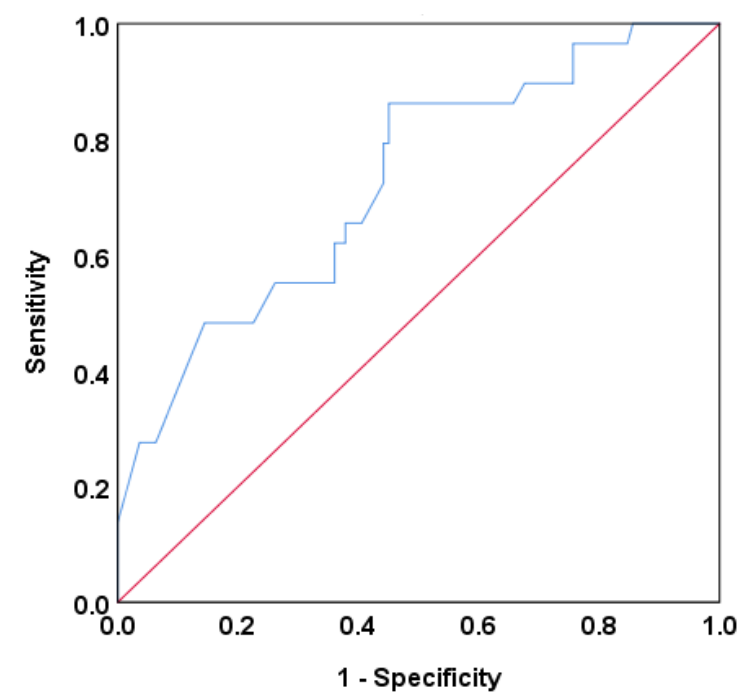

Fig. 3: ROC curve for $I^{131}$ therapy and complications are still problems plaguing the patients with DTC ${ }^{[10]}$. DTC frequently occurs in middleaged women and children, and its clinical manifestation is single thyroid nodules with hard texture. Enlargement of cervical lymph nodes is the first sign in about $10 \%$ of cases. Despite progress in the treatment of DTC recently, including surgery, radioactive iodine therapy and endocrine therapy using levothyroxine, the overall survival rate of patients with DTC is still far from satisfactory ${ }^{[11]}$. ${ }^{131}$ therapy has become the preferred clinical treatment method for DTC, which can well improve the prognosis of disease. $\mathrm{I}^{131}$, an isotope of iodine, is a radiopharmaceutical, and it will be absorbed by the stomach and intestines into the blood after oral administration. Then the $\beta$ rays emitted by it are used to shrink the swollen thyroid gland, thereby exerting a therapeutic effect, with little impact on surrounding tissues. However, the high incidence of hypothyroidism after $\mathrm{I}^{131}$ therapy is a problem urgently to be solved ${ }^{[12]}$. Due to the particularity of disease treatment, patients will suffer from anxiety and depression, affecting QOL. At present, the correlation between thyroid hormone levels and QOL after postoperative $\mathrm{I}^{131}$ therapy for DTC has been rarely studied. In the present study, such a correlation was explored, and the impact of hypothyroidism after postoperative $\mathrm{I}^{131}$ therapy on QOL of DTC patients was investigated.

DTC patients with different pathological bases have different incidence rates of hypothyroidism after postoperative $\mathrm{I}^{131}$ therapy. The clinical manifestations of patients vary, and the treatment effect is also greatly different. It is of important clinical significance to find out the influencing factors for the efficacy and overall prognosis of DTC patients after surgery, besides individual physical differences. The effects of gender, thyroid autoantibody levels, age and replacement therapy dose on hypothyroidism in the treatment of Graves' disease with radioactive $I^{131}$ have been evaluated ${ }^{[13]}$. Riley et al. ${ }^{[14]}$ studied the impacts of gender, age, course of disease, thyroid quality and

TABLE 4: CORRELATIONS BETWEEN INDICES OF THYROID FUNCTION AND QOL

\begin{tabular}{|c|c|c|c|c|c|c|c|c|c|c|}
\hline \multirow[t]{2}{*}{ Item } & \multicolumn{2}{|c|}{ TT3 (nmol/l) } & \multicolumn{2}{|c|}{ FT3 (pmol/l) } & \multicolumn{2}{|c|}{ FT4 (pmol/l) } & \multicolumn{2}{|c|}{ TT4 (nmol/l) } & \multicolumn{2}{|c|}{ TSH (mU/l) } \\
\hline & $r$ & $\mathrm{p}$ & $\mathbf{r}$ & p & $r$ & $\mathrm{p}$ & $r$ & $\mathrm{p}$ & $\mathbf{r}$ & p \\
\hline Physical function & 0.163 & 0.008 & 0.355 & 0.000 & 0.284 & 0.019 & 0.437 & 0.031 & 0.396 & 0.000 \\
\hline Role physical & 0.071 & 0.624 & 0.024 & 0.231 & 0.003 & 0.387 & 0.056 & 0.720 & 0.341 & 0.265 \\
\hline Bodily pain & 0.562 & 0.006 & 0.042 & 0.003 & 0.509 & 0.018 & 0.021 & 0.032 & 0.624 & 0.002 \\
\hline General health & 0.008 & 0.777 & 0.074 & 0.053 & 0.168 & 0.813 & 0.082 & 0.056 & 0.096 & 0.051 \\
\hline Vitality & 0.365 & 0.102 & 0.087 & 0.059 & 0.274 & 0.762 & 0.098 & 0.067 & 0.138 & 0.058 \\
\hline Social function & 0.675 & 0.008 & 0.661 & 0.018 & 0.690 & 0.021 & 0.703 & 0.005 & 0.362 & 0.008 \\
\hline Role emotional & 0.049 & 0.132 & 0.253 & 0.064 & 0.042 & 0.535 & 0.118 & 0.614 & 0.339 & 0.165 \\
\hline Mental health & 0.820 & 0.001 & 0.587 & 0.000 & 0.516 & 0.018 & 0.729 & 0.033 & 0.621 & 0.007 \\
\hline
\end{tabular}


$\mathrm{I}^{131}$ dose on the incidence rate of hypothyroidism after $I^{131}$ therapy, and found that age, course of disease, thyroid quality and $\mathrm{I}^{131}$ dose were all factors affecting the incidence rate of hypothyroidism. In this study, the incidence rate of hypothyroidism was $35.00 \%$ $(49 / 140)$ in 140 DTC patients undergoing postoperative $\mathrm{I}^{131}$ therapy. The results of univariate analysis revealed that the efficacy against hypothyroidism was related to the thyroid quality, course of disease, cumulative dose of $\mathrm{I}^{131}$ and operation method, and the differences were statistically significant $(\mathrm{p}<0.01)$. Furthermore, the influencing factors were incorporated into the multivariate logistic regression model. It was found that the thyroid quality, course of disease, cumulative dose of $\mathrm{I}^{131}$ and operation method were independent risk factors for hypothyroidism after postoperative $\mathrm{I}^{131}$ therapy, similar to the conclusion of the above studies.

In recent years, people's living standards have kept rising, and both health concepts and medical models have also changed. People have begun to pay more attention not only to survival time, but also to QOL. Whether QOL of thyroid cancer patients is different from that of normal people is a matter of interest in the medical field. To provide reliable clinical information for the hospitalization management and early psychological intervention of patients with thyroid carcinoma, it is necessary to study the influence of radioactive iodine therapy on QOL of patients. Daniel et al. ${ }^{[15]}$ found that $\mathrm{I}^{131}$ therapy after surgical resection could greatly improve QOL of patients. In this study, QOL of patients was compared before and after $\mathrm{I}^{131}$ therapy according to the SF-36 questionnaire. It was found that role physical, physical function, bodily pain, social function and mental health were all significantly superior after $\mathrm{I}^{131}$ therapy to those before $\mathrm{I}^{131}$ therapy, indicating that $\mathrm{I}^{131}$ therapy has a good curative effect. Considering the fact that liver dysfunction may occur in DTC patients after operation due to thyroid hormone levels affecting the health of patients, AI Hassan et al.

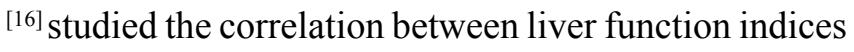
and serum indices of thyroid function. In this study, the correlation between thyroid function indices and QOL was further analyzed, and it was found that QOL indices physical function, bodily pain, social function and mental health were positively correlated with thyroid function indices TT3, FT3, FT4, TT4 and TSH ( $<<0.05$ or $\mathrm{p}<0.01$ ). Many scholars, strictly in accordance with the guidelines of the American Thyroid Association in 2015, take TSH as a screening index for DTC. Patients are usually grouped based on the postoperative TSH level, and then the changes in hypothyroidism and liver damage are compared ${ }^{[17]}$. However, the cutoff value of TSH level in predicting tumor progression or recurrence has not been clarified yet currently. In this study, therefore, ROC curve analysis was conducted for the TSH level according to the guidelines of the American Thyroid Association. The results showed that the optimal cutoff point of TSH level was 0.63 $\mathrm{mU} / 1$, that is, TSH at $0.63 \mathrm{mU} / 1$ has a better predictive value for QOL. Besides, the TSH level in DTC patients was observed after $\mathrm{I}^{131}$ therapy, and its correlation with $\mathrm{I}^{131}$ therapy was confirmed. At present, a fixed dose is mostly adopted in research based on the differentiated thyroid carcinoma guidelines ${ }^{[18]}$, which has defects in guiding the efficacy of $\mathrm{I}^{131}$ therapy. For this reason, the cutoff value of $\mathrm{I}^{131}$ dose was explored in this study. The results manifested that the cutoff value of $\mathrm{I}^{131}$ dose was $7.35 \mathrm{GBq}$, suggesting that $\mathrm{I}^{131}$ therapy at a dose of 7.35 GBq has a better therapeutic effect on DTC patients.

In conclusion, many influencing factors should be considered in the $\mathrm{I}^{131}$ therapy of hypothyroidism in DTC patients. QOL of patients will be affected by hypothyroidism after $\mathrm{I}^{131}$ therapy. Determining the appropriate level of TSH for prediction and the appropriate therapeutic dose of $\mathrm{I}^{131}$ for controlling TSH level is conducive to the improvement of QOL of patients.

\section{REFERENCES}

1. Lin CY, Chang JS, Huang SM, Hung CJ, Hung CL, Chang CT, et al. Experience of sorafenib treatment in differentiated thyroid cancer from Taiwan. J Formosan Med Assoc 2021;120(1):18995.

2. Haugen BR, Alexander EK, Bible KC, Doherty GM, Mandel SJ, Nikiforov YE. The American Thyroid Association Guidelines Task Force on Thyroid Nodules and Differentiated Thyroid Cancer. 2015 American Thyroid Association management guidelines for adult patients with thyroid nodules and differentiated thyroid cancer. Thyroid 2016;26(1):1-33.

3. Chopra S, Garg A, Ballal S, Bal CS. Lung metastases from differentiated thyroid carcinoma: prognostic factors related to remission and disease-free survival. Clin Endocrinol 2015;82(3):445-52.

4. Haugen BR. 2015 American Thyroid Association management guidelines for adult patients with thyroid nodules and differentiated thyroid cancer: what is new and what has changed? Cancer 2017;123(3):372-81.

5. Bano A, Chaker L, Plompen EP, Hofman A, Dehghan A, Franco $\mathrm{OH}$, Janssen HL, Murad SD, Peeters RP. Thyroid function and the risk of nonalcoholic fatty liver disease: the Rotterdam Study. J Clin Endocrinol Metabol 2016;101(8):3204-11.

6. Tseng YH, Huang YH, Lin TK, Wu SM, Chi HC, Tsai CY, et al. Thyroid hormone suppresses expression of stathmin and associated tumor growth in hepatocellular carcinoma. Sci Rep 2016;6:38756.

7. Hedman C, Djärv T, Strang P, Lundgren CI. Fear of recurrence and view of life affect health-related quality of life in patients 
with differentiated thyroid carcinoma: a prospective Swedish population-based study. Thyroid 2018;28(12):1609-17.

8. Giusti M, Gay S, Conte L, Cecoli F, Mortara L, Vera L, et al. Evaluation of Quality of Life in Patients with Differentiated Thyroid Cancer by Means of the Thyroid-Specific PatientReported Outcome Questionnaire: A 5-Year Longitudinal Study. Eur Thyroid J 2019;8(6):314-22.

9. Dong AS, Zhu LL, Shi BW, Zhou AY, Chen QQ, Guo WJ. Evaluation on the Applicability of the Chinese Version of SF -36 v2 Scale in Assessing the Quality of Life of Patients with Atrial Fibrillation. Chin General Pract 2015;3211-5.

10. Kiyota N, Robinson B, Shah M, Hoff AO, Taylor M, Li D, et al. 2864 Defining 131I-refractory differentiated thyroid cancer: efficacy and safety of lenvatinib by 131I-refractory criteria in the SELECT trial. Eur J Cancer 2015;51:S578.

11. Ortega AJ, Vázquez RG, Cuenca JI, Brocca MA, Castilla J, Martínez JM, et al. 131I treatment in Differentiated Thyroid Cancer and End-Stage Renal Disease. Spanish J Nuclear Med Molecular Imag 2016;35(1):29-33.

12. Karasawa K, Nihei K, Tanaka H, Shimizuguchi T. Treatment results of well-differentiated thyroid cancer patients with bone metastases treated with Iodine-131. Japanese J Head Neck Cancer 2014;40(1):93-7.

13. Nahar K, Akhter P. Factors Affecting and Outcome of
Radioactive Iodine Therapy in Hyperthyroidism: A study at Institute of Nuclear Medicine \& Allied Sciences (INMAS) Sylhet. Bangladesh J Nuclear Med 2016;19(1):19-23.

14. Riley AS, McKenzie GA, Green V, Schettino G, England RJ, Greenman J. The effect of radioiodine treatment on the diseased thyroid gland. Int J Radiation Biol 2019;95(12):171827.

15. Daniel FI, Lima LD, Grando LJ, Castro R, Cordeiro EA, Dos Santos CR. Salivary evaluation in radioactive I131 treated patients with thyroid carcinoma. Acta Odont Scand 2018;76(2):148-52.

16. Al Hassan MS, Saafan T, El Ansari W, Al Ansari AA, Zirie MA, Farghaly H, Abdelaal A. The largest reported papillary thyroid carcinoma arising in struma ovarii and metastasis to opposite ovary: case report and review of literature. Thyroid Res 2018;11(1):10.

17. Piantanida E, Ippolito S, Gallo D, Masiello E, Premoli P, Cusini C, Rosetti S, Sabatino J, Segato S, Trimarchi F, Bartalena L. The interplay between thyroid and liver: implications for clinical practice. J Endocrinol Invest 2020;43(7):885-99.

18. Padma S, Sundaram PS. Radioiodine as an adjuvant therapy and its role in follow-up of differentiated thyroid cancer. J Cancer Res Therapeutic 2016;12(3):1109-13. 